\title{
Traumatic Posterior Hip Dislocation In A 9 Year Child: A Case Report
}

\author{
KHANDGE AV ${ }^{1}$, SINGH HU ${ }^{2}$ \\ Dr. Ashwinkumar Vasant Khandge ${ }^{I}$ Specialty Medical Officer, Department of Orthopedics, T.N.M.C and B.Y.L \\ Nair Ch. Hospital Mumbai, Maharashtra, India. \\ Dr. Hemant Ugrasen Singh ${ }^{2}$ Specialty Medical Officer, Department of Orthopedics, L.T.M.M.C and L.T.M.G.H
}

\begin{abstract}
Traumatic hip dislocation in children is a relatively rare injury. If gone unnoticed and reduced later than six hours after injury it can have complications like avascular necrosis of the femoral head. We present a case of paediatric posterior hip dislocation: A nine-year-old female child with a history of fall from height (5 feet) following which patient suffered a right-sided posterior hip dislocation which was reduced within four hours of injury under general anesthesia followed by immobilization in a Thomas splint. The patient followed up after eight months with a completely normal hip.Closed reduction as rapidly as possible following the trauma or an open relocation of the dislocated hip under general anesthesia is the treatment recommended. As pain sensitivity in children differs from that in adults the examiner must always do a hip examination following a trauma to the lower limb in children. Hence, it is imperative for the examiner to assess the child holistically and not to exclude any hip joint condition without proper examination and accompanying radiology.
\end{abstract}

Keywords:- Traumatic; Children; Avascular Necrosis; General Anesthesia; Hip dislocation.

\section{INTRODUCTION}

Traumatic hip dislocation is rare in childhood. Therefore, much series consist a small number of patients in the literature [1-5].Owing to the elasticity of the peri articular structures in children with hip dislocation, associated acetabulum or femoral head fracture is rarer in comparison to adults [6]. Traumatic hip dislocation usually develops due to the following mechanisms: fall from height, fall of another person, road traffic accident [7]. The traumatic hip dislocation in children may be associated with avascular necrosis and post-traumatic arthritis if the reduction is delayed beyond six hours.Avascular necrosis is usually associated with a delay in reduction time beyond 6 hours [8].

Avascular necrosis of the femoral head in childhood may later lead on to an array of hip related problems in adulthood and traumatic hip dislocations should not be missed.

\section{CASE REPORT}

A nine-year-old female child presented with a history of fall from a height of eight feet followed by inability to walk and severe right-sided anterior hip joint pain. The child was brought to our ER within two hours and assessed for hip joint examination and radiological investigations which showed the hip to be in flexion, adduction and internal rotation. The diagnosis of a traumatic posterior hip joint dislocation was confirmed on x-rays: Fig. 1 and 2. The child was promptly evaluated for an emergency anesthetic fitness and under general anesthesia and fluoroscopy guidance the hip joint was reduced within five hours of injury: Fig. 3. The right hip joint the following reduction was promptly immobilized by applying a Thomas splint and admitted for observation. Post op x-rays should right femoral head well reduced without any obvious osteochondral fragment. The child was later discharged on the Thomas splint and weekly radiographs were taken for another four weeks. Following four weeks the Thomas splint was discontinued and Physiotherapy started. The child attained a full hip range of motion within two months and at twelve months' post trauma: Fig. 4 and 5, the radiographs did not show any signs of avascular necrosis of the femoral head and a Harris hip score of 92. 


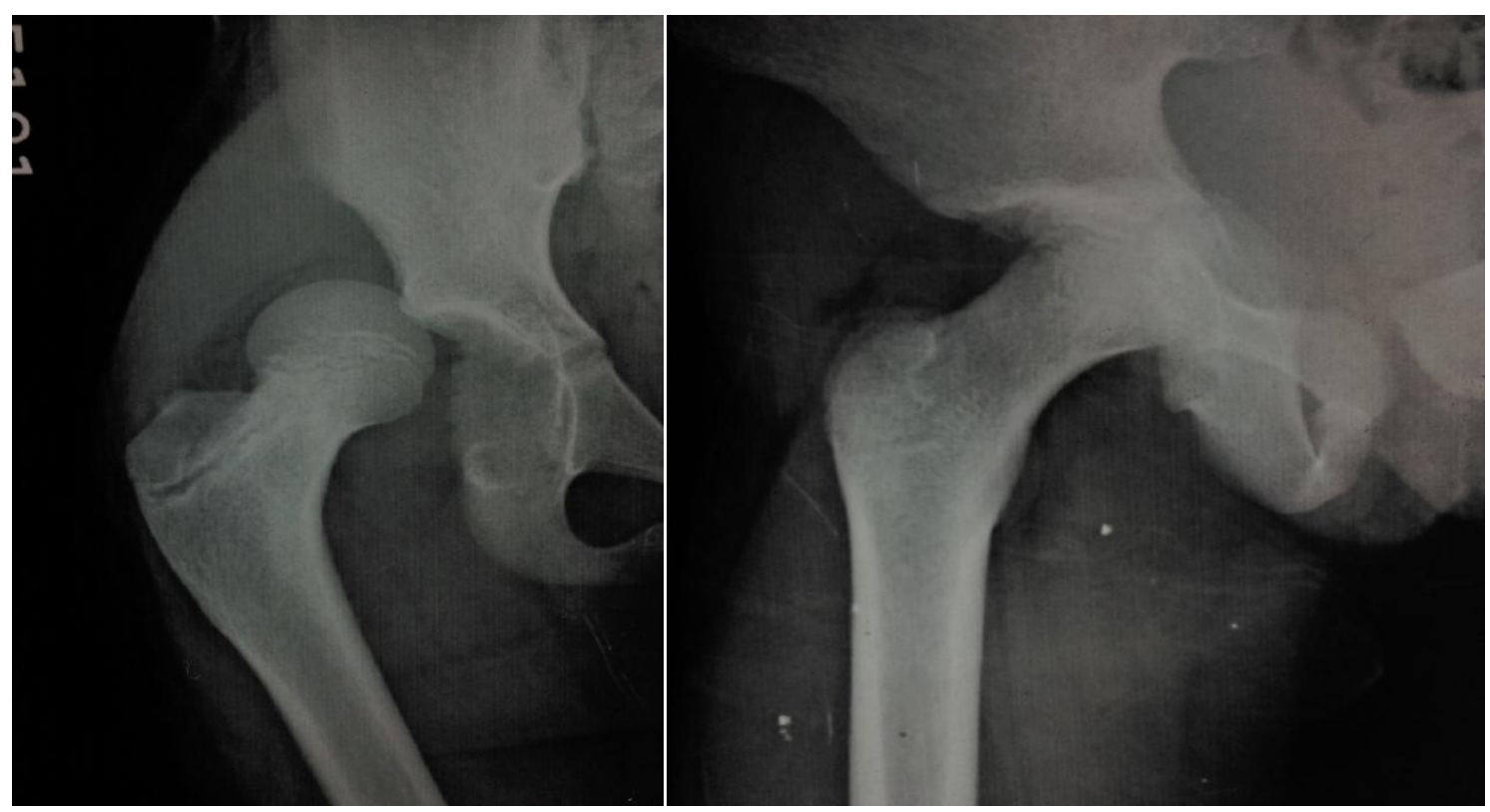

Figure 1 and 2: Anteroposterior $x$-ray and lateral $x$-ray radiographs of the right hip at presentation

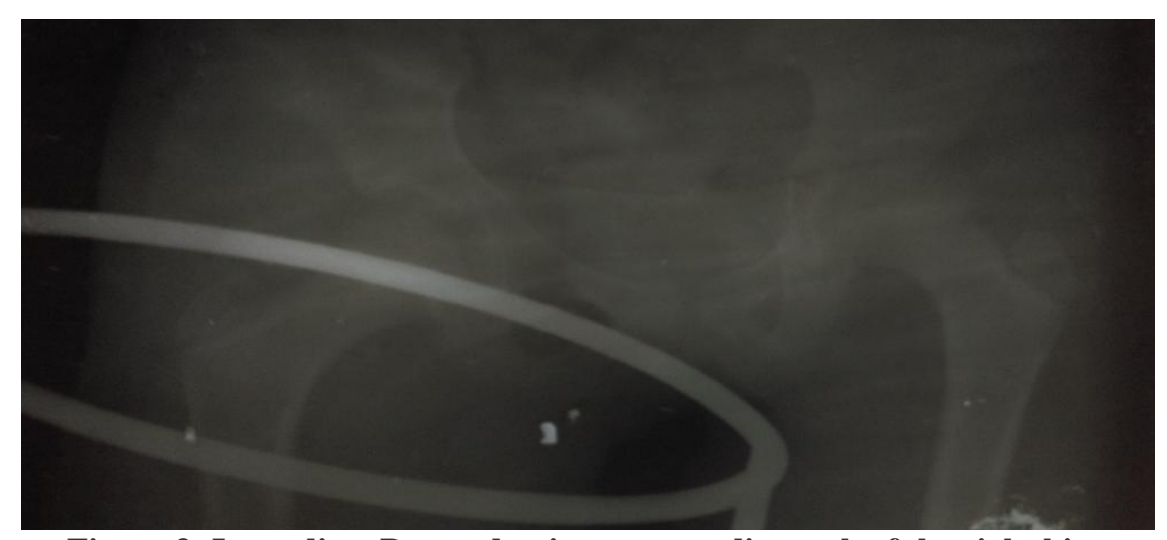

Figure 3: Immediate Post reduction x-ray radiograph of the right hip.

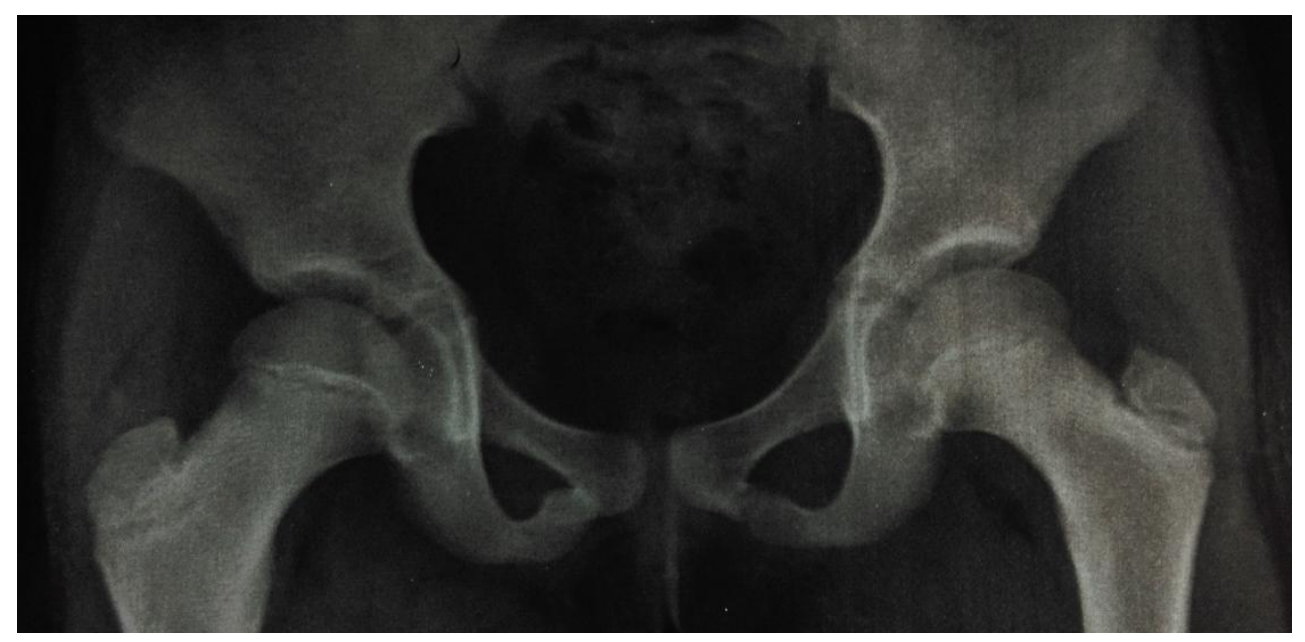

Figure 4: Four months' post reduction x-ray radiograph of the right hip joint 


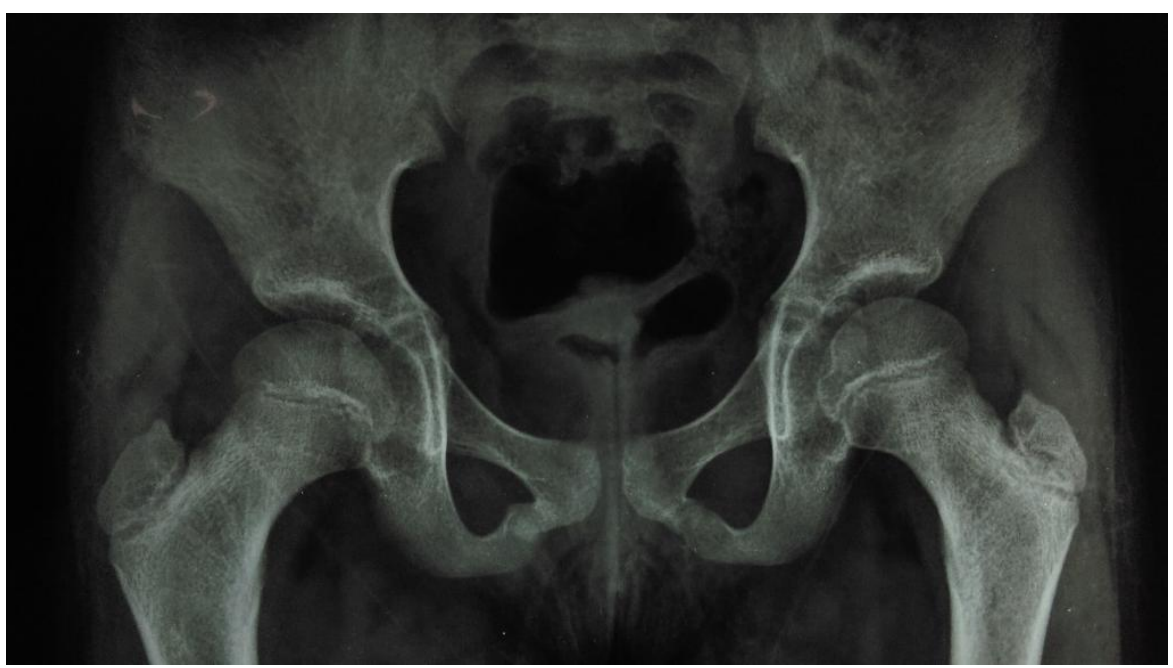

Figure 5: Twelvemonths' post reduction x-ray radiographs of the right hip joint.

\section{DISCUSSION}

Hip dislocation in the paediatric age group is a rare entity. It is a case of true orthopedic emergency and delay in diagnosis and management by the physician can have a devastating complication of avascular necrosis and consequent early onset arthritis of the respective hip joint. The risk of posterior hip dislocation after a trivial trauma is greater in the paediatric age group due to the laxity of the joint capsule and the peri-articular structures. Whereas the adolescents have a history of a relatively major trauma. Theusual mechanism of trauma is an axial load on a flexed and adducted hip [8].

Delay in identifying and treating a case of hip dislocation in paediatric age more than six hours after the injury increases the chances of avascular necrosis of the femoral head by around 20-times and its subsequent early onset osteoarthritis [9].Hence is necessary to evaluate all patients presenting to the Emergency Room with hip pain, inability to bear weight following a history of trauma. Following the clinical examination, a standard set of radiographs comprising of an anteroposterior and lateral radiographs is required to confirm the diagnosis and rule out any associated femoral head or acetabular fractures. It is thus imperative to get a rapid anesthetic evaluation of the child done and reduce the hip joint under sedation or general anesthesia.Checking of reduction by a palpable clunk and a post reduction intraoperative fluoroscopic evaluation is beneficial to confirm the joint reduction and the stability of the reduction. Immediately following the reduction, the apparent shortening and flexion of the involved joint disappears and both the limbs appear symmetrical. The Joint then should be immobilized either in a hip Spica cast or a Thomas splint or a skin traction device for 4-6 weeks.

Good results are obtained if the reduction is performed within 6 hours of injury. A long-term follow-up of usually more than 2 years is required to evaluate complications like avascular necrosis [9].

\section{CONCLUSION}

Traumatic hip dislocation is a rare presentation in children and timely management within six hours reduces the chances of avascular necrosis of the femoral head and the accompanying complications. The examiner should be vigilant towards patients presenting with a flexed posture of the hip with an inability to bear weight. Timely diagnosis and prompt management are the keystones to a successful outcome.

Conflictsofinterest

\section{REFERENCES}

[1] Kutty S, Thornes B, Curtin WA, Gilmore MF. Traumatic posterior dislocation of hip in children. Pediatr Emerg Care 2001;17:32-5. CrossRef

[2] Gürkan V, Dursun M, Orhun H, Sever GB, Kiliçkap C, Berkel T. Evalu- ation of pediatric patients with traumatic hip dislocation. Acta Orthop Traumatol Turc 2006;40:392-5.

[3] Zrig M, Mnif H, Koubaa M, Abid A. Traumatic hip dislocation in chil- dren. Acta Orthop Belg 2009;75:328-33.

[4] Kural C, Bayraktar K, Ertürk A, Ertürk H. Çocuklarda travmatik kalça çıkığı: iki olgu sunumu. Joint Dis Rel Surg 2006;17:49-52

[5] Freeman GE Jr. Traumatic dislocation of the hip in children: A report of 7 cases and review of the literature. J Bone Joint Surg Am 1961;43:401-6.

[6] Salisbury RD, Eastwood DM. Traumatic dislocation of the hip in chil- dren. Clin Orthop Relat Res 2000;377:106-11. 
[7] Barquet A. A vascular necrosis following traumatic hip dislocation in childhood: factors of influence. Acta Orthop Scand 1982;53:809-13.

[8] Herrera-Soto, J. A. and C. T. Price (2009). "Traumatic hip dislocations in children and adolescents: pitfalls and complications." J Am Acad Orthop Surg17(1): 15-21.

[9] Mehlman, C. T., et al. (2000). "Traumatic hip dislocation in children. Long-term followup of 42 patients." Clin Orthop Relat Res (376): 68-79. 